\title{
CHAOS AND SHADOWING AROUND A HOMOCLINIC TUBE
}

\author{
YANGUANG (CHARLES) LI
}

Received 7 February 2003

Let $F$ be a $C^{3}$ diffeomorphism on a Banach space $B . F$ has a homoclinic tube asymptotic to an invariant manifold. Around the homoclinic tube, Bernoulli shift dynamics of submanifolds is established through a shadowing lemma. This work removes an uncheckable condition of Silnikov (1968). Also, the result of Silnikov does not imply Bernoulli shift dynamics of a single map, but rather only provides a labeling of all invariant tubes around the homoclinic tube. The work of Silnikov was done in $\mathbb{R}^{n}$ and the current work is done in a Banach space.

\section{Introduction}

In [4], Silnikov introduced the concept of a homoclinic tube which can be obtained through a transversal intersection of the center-unstable and center-stable manifolds of a normally hyperbolic invariant manifold under a map in $\mathbb{R}^{n}$. Intuitively speaking, a homoclinic tube can be regarded as a homoclinic orbit on which points are replaced by submanifolds. Under a certain assumption [4, equation (11), page 625] which is uncheckable, all the invariant tubes in the neighborhood of the homoclinic tube can be labeled symbolically. Such a symbolic labeling does not imply Bernoulli shift of a single map. The result was proved through a contraction map argument on a sequence of metric spaces. In the current paper, we adopt a different approach developed in [1]. We will establish Bernoulli shift dynamics of submanifolds through a shadowing lemma. The uncheckable assumption of Silnikov is removed. We will work in a Banach space, while Silnikov worked in $\mathbb{R}^{n}$.

Especially for high-dimensional systems, homoclinic tubes are more dominant structures than homoclinic orbits. In fact, the invariant manifold that the homoclinic tube is asymptotic to, can contain smaller scale chaotic dynamics as discussed in $[2,3]$. Although structures in a neighborhood of a homoclinic 
orbit have been extensively and intensively investigated, structures around a homoclinic tube have not been well studied $[2,3,4]$. We believe that homoclinic tubes will play an important role in the theory of chaos in Hamiltonian partial differential equations.

The paper is organized as follows: Section 2 contains the setup and definitions, Section 3 deals with Fenichel fiber coordinates and a $\lambda$-lemma, and Section 4 deals with shadowing lemma and chaos.

\section{The setup and definitions}

The setup is as follows.

(A1) Let $B$ be a Banach space on which a $C^{3}$ diffeomorphism $F$ is defined. There is a normally (transversally) hyperbolic invariant $C^{3}$ submanifold $S$. Let $W^{\text {cu }}$ and $W^{\text {cs }}$ be the $C^{3}$ center-unstable and center-stable manifolds of $S$. There exist a $C^{2}$ invariant family of $C^{3}$ unstable Fenichel fibers $\left\{\mathscr{F}^{u}(q): q \in S\right\}$ and a $C^{2}$ invariant family of $C^{3}$ stable Fenichel fibers $\left\{\mathscr{F}^{s}(q): q \in S\right\}$ inside $W^{\text {cu }}$ and $W^{\text {cs }}$, respectively, such that

$$
W^{\mathrm{cu}}=\bigcup_{q \in S} \mathscr{F}^{u}(q), \quad W^{\mathrm{cs}}=\bigcup_{q \in S} \mathscr{F}^{s}(q)
$$

There are positive constants $\kappa$ and $C$ such that

$$
\begin{aligned}
& \left\|F^{-n}\left(q^{-}\right)-F^{-n}(q)\right\| \leq C e^{-\kappa n}\left\|q^{-}-q\right\|, \quad \forall n \in \mathbb{Z}^{+}, \forall q \in S \forall q^{-} \in \mathscr{F}^{u}(q), \\
& \left\|F^{n}\left(q^{+}\right)-F^{n}(q)\right\| \leq C e^{-\kappa n}\left\|q^{+}-q\right\|, \quad \forall n \in \mathbb{Z}^{+}, \forall q \in S \forall q^{+} \in \mathscr{F}^{s}(q), \\
& \left\|F^{n}\left(q_{1}\right)-F^{n}\left(q_{2}\right)\right\| \leq C e^{\kappa_{1}|n|}\left\|q_{1}-q_{2}\right\|, \quad \forall n \in \mathbb{Z} \forall q_{1}, q_{2} \in S,
\end{aligned}
$$

where $\kappa_{1} \ll \kappa$; for example, $\kappa_{1}<\kappa / 300$. $W^{\text {cu }}$ and $W^{\text {cs }}$ intersect along an isolated transversal homoclinic tube $\xi$ asymptotic to $S$,

$$
\xi=\left(\cdots S_{-1} S_{0} S_{1} \cdots\right) \text {, }
$$

where $S_{j}=F^{j} S_{0}$, for all $j \in \mathbb{Z}$, and $S_{0}$ is $C^{3}$. For all $j \in \mathbb{Z}$ and for all $q_{j} \in S_{j}, q_{j}$ is on a unique stable fiber $\mathscr{F}^{s}\left(q_{+}\right), q_{+} \in S$ and a unique unstable fiber $\mathscr{F}^{u}\left(q_{-}\right), q_{-} \in$ $S$. We denote such correspondences by $\varphi_{j}^{+}$and $\varphi_{j}^{-}$, respectively, where $\varphi_{j}^{ \pm}(S)=$ $S_{j} . \varphi_{j}^{ \pm}$are $C^{2}$ diffeomorphisms. Let

$$
\left\|\varphi_{j}^{ \pm}-\mathrm{id}\right\|_{C^{1}}=\sup _{q \in S}\left\{\max \left\{\left\|\varphi_{j}^{ \pm}(q)-q\right\|,\left\|D \varphi_{j}^{ \pm}(q)-\mathrm{id}\right\|\right\}\right\}
$$

where id is the identity map and $D \varphi_{j}^{ \pm}$denotes the differential of $\varphi_{j}^{ \pm}$. As $j \rightarrow+\infty$,

$$
\left\|\varphi_{j}^{+}-\mathrm{id}\right\|_{C^{1}} \longrightarrow 0
$$


As $j \rightarrow-\infty$,

$$
\left\|\varphi_{j}^{-}-\mathrm{id}\right\|_{C^{1}} \longrightarrow 0
$$

Let

$$
\begin{array}{r}
\theta=\inf _{u, v, w, q_{j} \in S_{j}, j \in \mathbb{Z}}\left\{\min \{\|u-v\|,\|v-w\|,\|w-u\|\} \mid u \in T_{q_{j}} \mathscr{F}^{u}\left(q_{-}\right),\right. \\
\left.v \in T_{q_{j}} \mathscr{F}^{s}\left(q_{+}\right), w \in T_{q_{j}} S_{j},\|u\|=\|v\|=\|w\|=1\right\},
\end{array}
$$

where $T_{q_{j}}$ indicates the tangent space at $q_{j}$. In this paper, transversality always implies that such angle $\theta$ is positive.

(A2) Let $\Omega$ be a neighborhood of $S$. Then there exists a $J>0$ such that $S_{j} \subset \Omega$ for all $|j| \geq J$. Let

$$
d=\inf _{q \in S_{j} \cup S,|j| \geq J}\{\text { distance }\{q, \partial \Omega\}\},
$$

then $d>0$. Let $\Omega_{j}$ be a neighborhood of $S_{j}$, for all $|j|<J$,

$$
d_{j}=\inf _{q \in S_{j}}\left\{\text { distance }\left\{q, \partial \Omega_{j}\right\}\right\},
$$

then $d_{j}>0$. The collection $B_{\xi}=\left\{\Omega, \Omega_{j}|j|<J\right\}$ is called a tubular neighborhood of $\xi \cup S$. For any $0<n<\infty$, there exists such a tubular neighborhood $B \xi$ of $\xi \cup S$ such that for any $q_{1} \in B_{\xi}$ there is a $q \in \xi \cup S, q_{1}$ and $q$ belong to the same $\Omega$ or $\Omega_{j}|j|<J$,

$$
\left\|D^{\ell} F^{ \pm n}\left(q_{1}\right)-D^{\ell} F^{ \pm n}(q)\right\|<1 \quad(\ell=1,2) .
$$

Moreover,

$$
\max _{+,-} \sup _{q \in \xi \cup S}\left\|D^{2} F^{ \pm n}(q)\right\|<\infty .
$$

Let

$$
\Lambda_{\ell}=\max _{+,-} \sup _{q \in B_{\xi}}\left\|D^{\ell} F^{ \pm n}(q)\right\|<\infty \quad(\ell=1,2) .
$$

If $\xi \cup S$ is compact, then assumption (A2) holds [1]. Next we will introduce a pseudoinvariant tube. An invariant tube is a sequence of submanifolds $\left\{B_{j}\right\}_{j \in \mathbb{Z}}$ such that

$$
F\left(B_{j}\right)=B_{j+1}, \quad F^{-1}\left(B_{j}\right)=B_{j-1}, \quad \forall j \in \mathbb{Z} .
$$


926 Chaos and shadowing around a homoclinic tube

Definition 2.1. Segment-0 is defined as the finite sequence of $(2 N+1) S$ 's

$$
\eta_{0}=(S \cdots S)
$$

and segment- 1 is defined as the finite sequence

$$
\eta_{1}=\left(S_{-N} S_{-N+1} \cdots S_{0} \cdots S_{N-1} S_{N}\right)
$$

where $N$ is a large positive integer.

Definition 2.2. Let $\Sigma$ be a set that consists of elements of the doubly infinite sequence form

$$
a=\left(\cdots a_{-2} a_{-1} a_{0}, a_{1} a_{2} \cdots\right),
$$

where $a_{k} \in\{0,1\}, k \in \mathbb{Z}$. We introduce a topology in $\Sigma$ by taking as a neighborhood basis of

$$
a^{*}=\left(\cdots a_{-2}^{*} a_{-1}^{*} a_{0}^{*}, a_{1}^{*} a_{2}^{*} \cdots\right)
$$

the set

$$
\mathscr{A}_{j}=\left\{a \in \Sigma \mid a_{k}=a_{k}^{*}(|k|<j)\right\}
$$

for $j=1,2, \ldots$. This makes $\Sigma$ a topological space. The Bernoulli shift automorphism $\chi$ is defined on $\Sigma$ by

$$
\chi: \Sigma \longmapsto \Sigma, \quad \forall a \in \Sigma, \quad \chi(a)=b, \quad \text { where } b_{k}=a_{k+1}
$$

The Bernoulli shift automorphism $\chi$ exhibits sensitive dependence on initial conditions, which is a hallmark of chaos.

For any $\delta>0$, there exists $N>0$ such that

$$
\left\|\varphi_{j}^{+}-\mathrm{id}\right\|_{C^{1}}<\delta, \quad\left\|\varphi_{-j}^{-}-\mathrm{id}\right\|_{C^{1}}<\delta, \quad \forall j \geq N,
$$

by assumption (A1).

Definition 2.3. To each $a_{k} \in\{0,1\}$, we associate the segment- $a_{k}, \eta_{a_{k}}$. Then each doubly infinite sequence

$$
a=\left(\cdots a_{-2} a_{-1} a_{0}, a_{1} a_{2} \cdots\right)
$$

is associated with a $\delta$-pseudoinvariant tube

$$
\eta_{a}=\left(\cdots \eta_{a_{-2}} \eta_{a_{-1}} \eta_{a_{0}}, \eta_{a_{1}} \eta_{a_{2}} \cdots\right)
$$




\section{Fenichel fiber coordinates and a $\lambda$-lemma}

3.1. Fenichel fiber coordinates. We will introduce Fenichel fiber coordinates in a neighborhood of $S$. For any $\theta \in S$, let

$$
E^{u}(\theta)=T_{\theta} \mathscr{F}^{u}(\theta), \quad E^{c}(\theta)=T_{\theta} S, \quad E^{s}(\theta)=T_{\theta} \mathscr{F}^{s}(\theta)
$$

where $T_{\theta}$ indicates the tangent space at $\theta . E^{u}$ and $E^{s}$ provide a coordinate system for a neighborhood of $S$, that is, any point in this neighborhood has a unique coordinate

$$
\left(\tilde{v}^{s}, \tilde{\theta}, \tilde{v}^{u}\right), \quad \tilde{v}^{s} \in E^{s}(\tilde{\theta}), \tilde{v}^{u} \in E^{u}(\tilde{\theta}), \tilde{\theta} \in S
$$

Fenichel fibers provide another coordinate system for the neighborhood of $S$. For any $\theta \in S$, the Fenichel fibers $\mathscr{F}^{s}(\theta)$ and $\mathscr{F}^{u}(\theta)$ have the expressions

$$
\begin{aligned}
\tilde{v}^{s} & =v^{s}, \quad \tilde{v}^{u}=v^{u}, \\
\tilde{\theta} & =\theta+\Theta_{s}\left(v^{s}, \theta\right), \quad \tilde{\theta}=\theta+\Theta_{u}\left(v^{u}, \theta\right), \\
\tilde{v}^{u} & =V_{s}\left(v^{s}, \theta\right), \quad \tilde{v}^{s}=V_{u}\left(v^{u}, \theta\right),
\end{aligned}
$$

where $v^{s}$ and $v^{u}$ are the parameters parametrizing $\mathscr{F}^{s}(\theta)$ and $\mathscr{F}^{u}(\theta)$,

$$
\Theta_{z}(0, \theta)=\frac{\partial}{\partial v^{z}} \Theta(0, \theta)=V_{z}(0, \theta)=\frac{\partial}{\partial v^{z}} V_{z}(0, \theta)=0, \quad z=u, s
$$

and $\Theta_{z}\left(v^{z}, \theta\right)$ and $V_{z}\left(v^{z}, \theta\right)(z=u, s)$ are $C^{3}$ in $v^{z}$ and $C^{2}$ in $\theta$. The coordinate transformation from $\left(v^{s}, \theta, v^{u}\right)$ to $\left(\tilde{v}^{s}, \tilde{\theta}, \tilde{v}^{u}\right)$

$$
\begin{aligned}
\tilde{v}^{s} & =v^{s}+V_{u}\left(v^{u}, \theta\right), \\
\tilde{\theta} & =\theta+\Theta_{u}\left(v^{u}, \theta\right)+\Theta_{s}\left(v^{s}, \theta\right), \\
\tilde{v}^{u} & =v^{u}+V_{s}\left(v^{s}, \theta\right)
\end{aligned}
$$

is a $C^{2}$ diffeomorphism. In terms of the Fenichel coordinate $\left(v^{s}, \theta, v^{u}\right)$, the Fenichel fibers coincide with their tangent spaces. From now on, we always work with the Fenichel coordinate $\left(v^{s}, \theta, v^{u}\right)$.

3.2. $\lambda$-lemma. For all $j \in \mathbb{Z}$ and for all $q_{j} \in S_{j}, q_{j}$ is on a unique stable fiber $\mathscr{F}^{s}\left(q_{+}\right), q_{+} \in S$ and a unique unstable fiber $\mathscr{F}^{u} u\left(q_{-}\right), q_{-} \in S$. Let

$$
E^{u}\left(q_{j}\right)=T_{q_{j}} \mathscr{F}^{u}\left(q_{-}\right), \quad E^{c}\left(q_{j}\right)=T_{q_{j}} S_{j}, \quad E^{s}\left(q_{j}\right)=T_{q_{j}} \mathscr{F}^{s}\left(q_{+}\right)
$$

By assumption (A1), $E^{u}\left(q_{j}\right), E^{c}\left(q_{j}\right)$, and $E^{s}\left(q_{j}\right)$ are $C^{2}$ in $q_{j} \in S_{j}$. 
Lemma 3.1 ( $\lambda$-lemma). For any $\epsilon>0$, there exists a $J>0$ such that

(1) when $j \geq J, E^{u}\left(q_{j}\right) \oplus E^{c}\left(q_{j}\right)$ is $\epsilon$-close to $E^{u}\left(q_{+}\right) \oplus E^{c}\left(q_{+}\right)$;

(2) when $j \leq-J, E^{s}\left(q_{j}\right) \oplus E^{c}\left(q_{j}\right)$ is $\epsilon$-close to $E^{s}\left(q_{-}\right) \oplus E^{c}\left(q_{-}\right)$.

Proof. When $j(>0)$ is large enough, $q_{j}$ is in a neighborhood of $S$ where $\mathscr{F}^{s}\left(q_{+}\right)=$ $E^{s}\left(q_{+}\right)$. Let $v_{1} \in E^{u}\left(q_{j}\right) \oplus E^{c}\left(q_{j}\right),\left\|v_{1}\right\|=1$. One can represent $v_{1}$ in the frame $\left(E^{s}\left(q_{+}\right), E^{u}\left(q_{+}\right) \oplus E^{c}\left(q_{+}\right)\right)$,

$$
v_{1}=\left(v_{1}^{s}, v_{1}^{u c}\right)
$$

Let $\lambda_{1}=\left\|v_{1}^{s}\right\| /\left\|v_{1}^{u c}\right\|$. By assumption (A1), transversality implies that $\lambda_{1}$ has an absolute upper bound. The rest of the argument is the same as that in [1].

3.3. A rectification. Next we conduct a rectification in a neighborhood of $S$, which is necessary for graph transform argument later on. When $j(>0)$ is large enough, $q_{j}$ is in a neighborhood of $S$ where $\mathscr{F}^{s}\left(q_{+}\right)=E^{s}\left(q_{+}\right)$. Thus $E^{s}\left(q_{j}\right)=$ $E^{s}\left(q_{+}\right)$. For any $\tilde{v}^{u} \in E^{u}\left(q_{+}\right),\left\|\tilde{v}^{u}\right\|=1, \tilde{v}^{u}$ has the representation in the frame $\left(E^{s}\left(q_{j}\right), E^{u}\left(q_{j}\right) \oplus E^{c}\left(q_{j}\right)\right)$,

$$
\tilde{v}^{u}=v^{s}+v^{u c}
$$

All such $v^{u c}$ 's span the projection $\tilde{E}^{u}\left(q_{j}\right)$ of $E^{u}\left(q_{+}\right)$onto $E^{u}\left(q_{j}\right) \oplus E^{c}\left(q_{j}\right)$, where $E^{u}\left(q_{j}\right) \oplus E^{c}\left(q_{j}\right)$ is $C^{2}$ in $q_{j}$. Shifting $E^{u}\left(q_{+}\right), E^{c}\left(q_{+}\right)$, and $E^{s}\left(q_{+}\right)$to $q_{j}$, they are also $C^{2}$ in $q_{j}$. Representing $E^{u}\left(q_{j}\right) \oplus E^{c}\left(q_{j}\right)$ in the frame $\left(E^{u}\left(q_{+}\right), E^{c}\left(q_{+}\right), E^{s}\left(q_{+}\right)\right)$, $\tilde{E}^{u}\left(q_{j}\right)$ can be obtained from $E^{u}\left(q_{j}\right) \oplus E^{c}\left(q_{j}\right)$ by restricting $\theta=0$, where $\theta$ coordinatizes $E^{c}\left(q_{+}\right)$. Thus $\tilde{E}^{u}\left(q_{j}\right)$ is also $C^{2}$ in $q_{j}$. The rectification amounts to replacing $E^{u}\left(q_{j}\right)$ by $\tilde{E}^{u}\left(q_{j}\right)$. We will use the same notation $E^{u}\left(q_{j}\right)$. Similarly, when $j(>0)$ is large enough, one can rectify $E^{s}\left(q_{-j}\right)$ inside $E^{s}\left(q_{-j}\right) \oplus E^{c}\left(q_{-j}\right)$.

\section{Shadowing lemma and chaos}

Let $\eta_{a}$ be a $\delta$-pseudoinvariant tube defined in Definition 2.3,

$$
\eta_{a}=\left(\cdots \tilde{S}_{-1} \tilde{S}_{0} \tilde{S}_{1} \cdots\right)
$$

where $\tilde{S}_{j}=S_{k}$ or $S ; j, k \in \mathbb{Z}$. Denote by $\hat{E}$ the transversal bundle

$$
\widehat{E}=\left\{\left(q, E^{u}(q), E^{s}(q)\right) \mid q \in \eta_{a}\right\}
$$

which serves as a coordinate system around $\eta_{a}$ with the coordinate denoted by

$$
\left(q, x^{u}, x^{s}\right), \quad q \in \eta_{a}, x^{u} \in E^{u}(q), x^{s} \in E^{s}(q) .
$$


In this coordinate system, the map $F^{n}$ has the representation

$$
F^{n}\left(q, x^{u}, x^{s}\right)=\left(f\left(q, x^{u}, x^{s}\right), g^{u}\left(q, x^{u}, x^{s}\right), g^{s}\left(q, x^{u}, x^{s}\right)\right)
$$

where $n$ is a large positive integer. If $q \in \tilde{S}_{j}$, then $f\left(q, x^{u}, x^{s}\right) \in \tilde{S}_{j+n}$.

Lemma 4.1. For all $\mu>0$, fix an $n$ large enough and fix an $\epsilon$ small enough if $\delta$ is sufficiently small, then

$$
\begin{array}{llll}
\left(\Lambda_{1}\right)^{k} \Pi_{3}^{s}<\frac{1}{2} & (0 \leq k \leq 2), & \Pi_{\ell}^{s}<\mu & (\ell=1,2), \\
\left(\Lambda_{1}\right)^{k} \hat{\Pi}_{2}^{u}<\frac{1}{2} & (0 \leq k \leq 2), & \Pi_{\ell}^{u}<\mu & (\ell=1,3),
\end{array}
$$

where $\left\|x^{u}\right\| \leq \epsilon,\left\|x^{s}\right\| \leq \epsilon, D_{1}=D_{q}, D_{2}=D_{x^{u}}, D_{3}=D_{x^{s}}$, and

$$
\begin{aligned}
& \Pi_{\ell}^{s}=\sup _{q, x^{u}, x^{s}}\left\|D_{\ell} g^{s}\left(q, x^{u}, x^{s}\right)\right\| \quad(\ell=1,2,3), \\
& \Pi_{\ell}^{u}=\sup _{q, x^{u}, x^{s}}\left\|D_{\ell} g^{u}\left(q, x^{u}, x^{s}\right)\right\| \quad(\ell=1,2,3), \\
& \hat{\Pi}_{2}^{u}=\sup _{q, x^{u}, x^{s}}\left\|\left\{D_{2} g^{u}\left(q, x^{u}, x^{s}\right)\right\}^{-1}\right\| .
\end{aligned}
$$

Proof. The proof of this lemma follows from assumptions (A1) and (A2) and the fact that along segment- 0 and segment-1, the center-unstable and centerstable bundles $E^{u}(q) \oplus E^{c}(q)$ and $E^{s}(q) \oplus E^{c}(q)$ are invariant under the linearized flow [1].

Let $\Gamma_{\epsilon}$ be the space of sections of $\hat{E}$,

$$
\Gamma_{\epsilon}=\left\{\sigma \mid \sigma(q)=\left(q, x^{u}(q), x^{s}(q)\right), q \in \eta_{a},\left\|x^{u}(q)\right\| \leq \epsilon,\left\|x^{s}(q)\right\| \leq \epsilon\right\} .
$$

We define the $C^{0}$ norm of $\sigma \in \Gamma_{\epsilon}$ as

$$
\|\sigma\|_{C^{0}}=\max \left\{\sup _{q \in \eta_{a}}\left\|x^{u}(q)\right\|, \sup _{q \in \eta_{a}}\left\|x^{s}(q)\right\|\right\}
$$

Then we define a Lipschitz seminorm on $\Gamma_{\epsilon}$ as

$$
\operatorname{Lip}\{\sigma\}=\max \left\{\sup _{\left\|q_{1}-q_{2}\right\| \leq \Delta} \frac{\left\|x^{u}\left(q_{1}\right)-x^{u}\left(q_{2}\right)\right\|}{\left\|q_{1}-q_{2}\right\|}, \sup _{\left\|q_{1}-q_{2}\right\| \leq \Delta} \frac{\left\|x^{s}\left(q_{1}\right)-x^{s}\left(q_{2}\right)\right\|}{\left\|q_{1}-q_{2}\right\|}\right\}
$$


for some small fixed $\Delta>0$. Let $\Gamma_{\epsilon, \gamma}$ be a subset of $\Gamma_{\epsilon}$,

$$
\Gamma_{\epsilon, \gamma}=\left\{\sigma \in \Gamma_{\epsilon} \mid \operatorname{Lip}\{\sigma\} \leq \gamma\right\}
$$

For any $\sigma \in \Gamma_{\epsilon, \gamma}$,

$$
\sigma(q)=\left(q, x^{u}(q), x^{s}(q)\right), \quad q \in \eta_{a}
$$

we define the graph transform $G$ as follows:

$$
(G \sigma)(q)=\left(q, x_{1}^{u}(q), x_{1}^{s}(q)\right)
$$

where

$$
\begin{aligned}
f\left(q^{-}, x^{u}\left(q^{-}\right), x^{s}\left(q^{-}\right)\right) & =q, \\
g^{s}\left(q^{-}, x^{u}\left(q^{-}\right), x^{s}\left(q^{-}\right)\right) & =x_{1}^{s}(q), \\
f\left(q, x_{1}^{u}(q), x^{s}(q)\right) & =q^{+}, \\
g^{u}\left(q, x_{1}^{u}(q), x^{s}(q)\right) & =x^{u}\left(q^{+}\right),
\end{aligned}
$$

for some $q^{-}$and $q^{+}$.

TheOREM 4.2. The graph transform $G$ is a contraction map on $\Gamma_{\epsilon, \gamma}$. The graph of the fixed point $\sigma^{*}$ of $G$ is an invariant tube under $F$.

Proof. The proof of this theorem is similar to that given in detail in [1].

Graph $\left\{\sigma^{*}\right\}$ is the invariant tube that $\epsilon$-shadows the $\delta$-pseudoinvariant tube $\eta_{a}$. Let $S^{*}$ be the element of Graph $\left\{\sigma^{*}\right\}$ that shadows the midelement of $\eta_{a}$, which is either $S_{0}$ or $S$. Such $S^{*}$ 's for all $\eta_{a}$ form a set $\Xi$ of submanifolds. It is obvious that the following theorem holds.

Theorem 4.3 (chaos theorem). The set $\Xi$ of submanifolds is invariant under the map $F^{2 N+1}$. The action of $F^{2 N+1}$ on $\Xi$ is topologically conjugate to the action of the Bernoulli shift automorphism $\chi$ on $\Sigma$. That is, there exists a homeomorphism $\phi: \Sigma \mapsto \Xi$ such that the following diagram commutes:

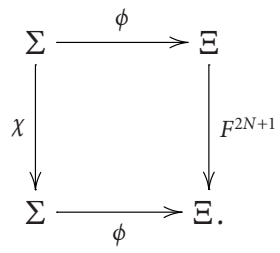




\section{References}

[1] Y. Li, Chaos and shadowing lemma for autonomous systems of infinite dimensions, submitted to Ann. Inst. H. Poincaré Anal. Non Linéaire.

[2] Homoclinic tubes in nonlinear Schrödinger equation under Hamiltonian perturbations, Progr. Theoret. Phys. 101 (1999), no. 3, 559-577.

[3] Homoclinic tubes in discrete nonlinear Schrödinger equation under Hamiltonian perturbations, Nonlinear Dynam. 31 (2003), no. 4, 393-434.

[4] L. P. Silnikov, Structure of the neighborhood of a homoclinic tube of an invariant torus, Soviet Math. Dokl. 9 (1968), no. 3, 624-628.

Yanguang (Charles) Li: Department of Mathematics, University of Missouri, Columbia, MO 65211, USA

E-mail address: cli@math.missouri.edu 


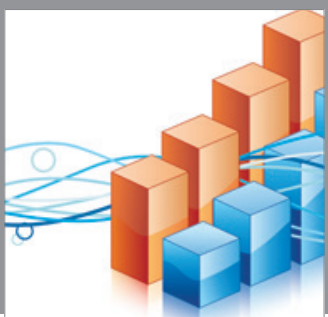

Advances in

Operations Research

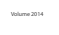

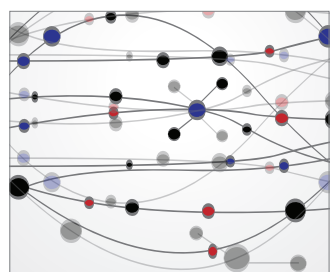

\section{The Scientific} World Journal
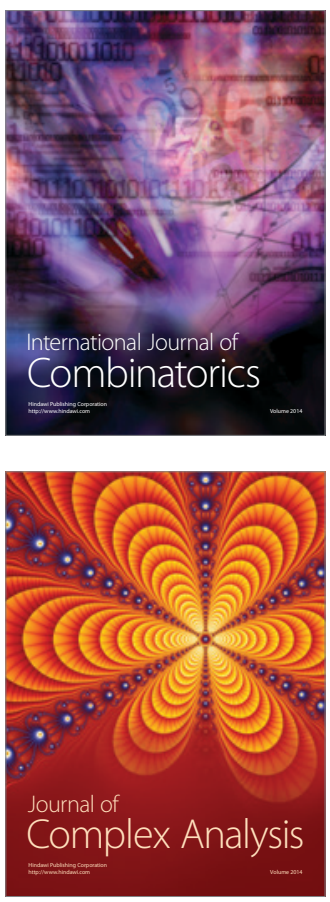

International Journal of

Mathematics and

Mathematical

Sciences
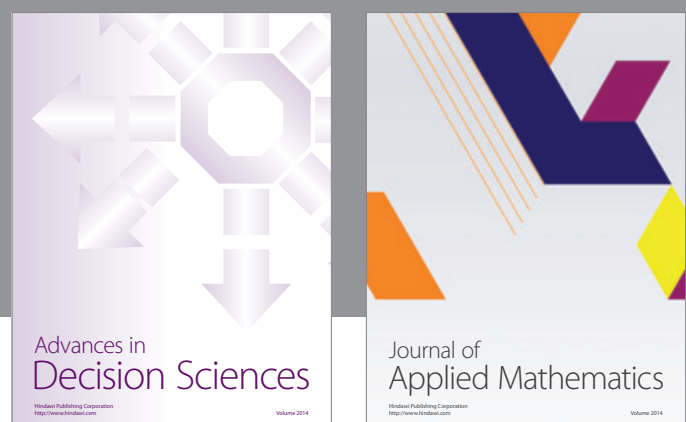

Journal of

Applied Mathematics
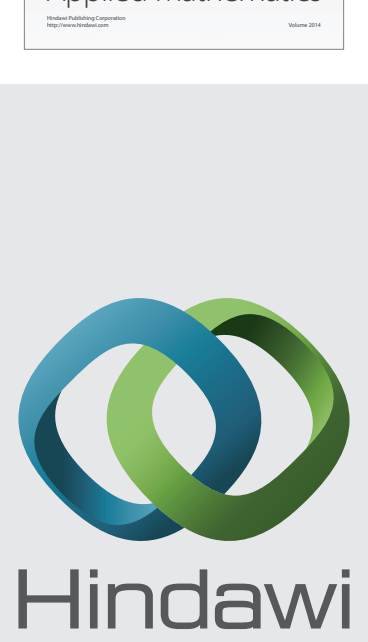

Submit your manuscripts at http://www.hindawi.com
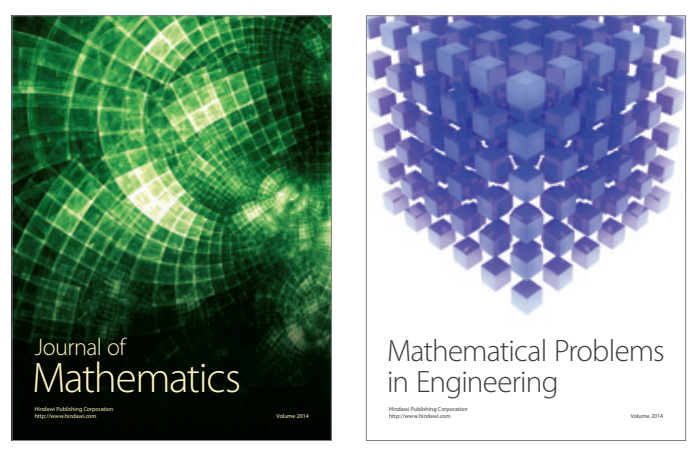

Mathematical Problems in Engineering
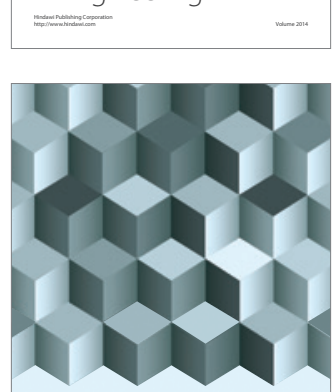

Journal of

Function Spaces
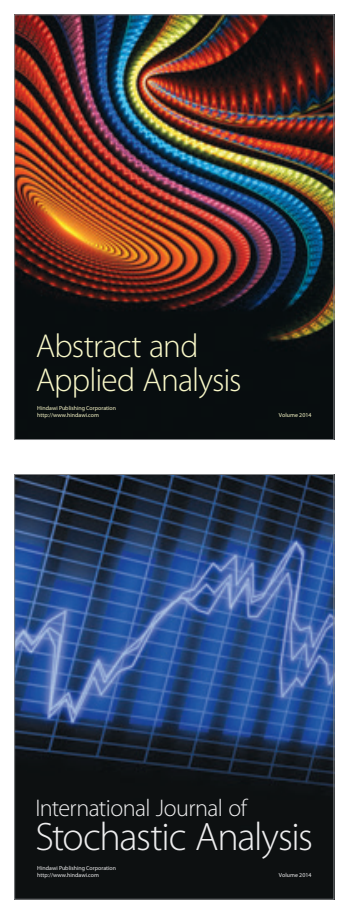

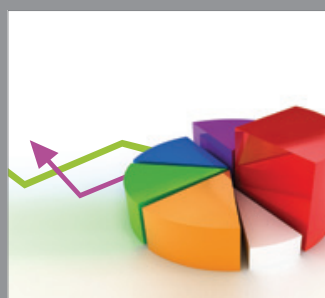

ournal of

Probability and Statistics

Promensencen
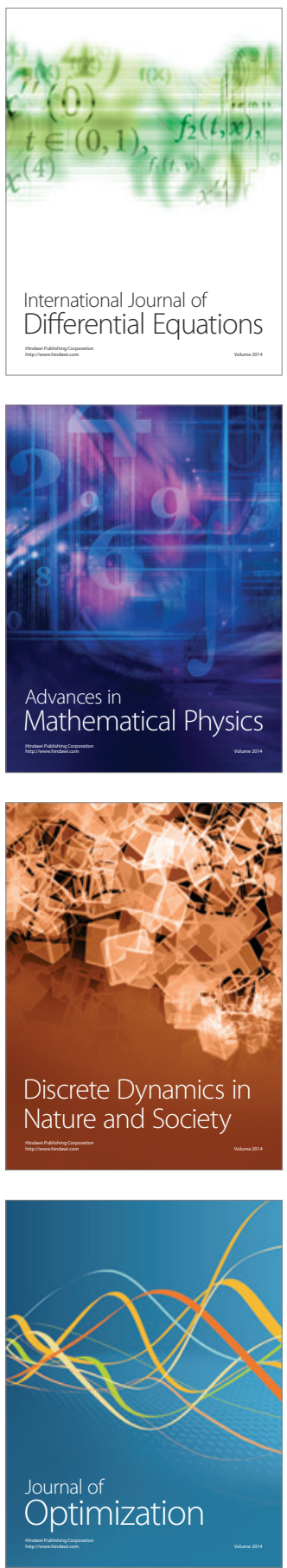